\title{
Melissa Wilde, Vatican II: a sociological analysis of religious change
}

Princeton, Princeton University Press, 2007, 224 p.

\section{François Weiser}

\section{(2) OpenEdition}

\section{Journals}

Édition électronique

URL : http://journals.openedition.org/assr/22097

DOI : $10.4000 /$ assr.22097

ISSN : $1777-5825$

Éditeur

Éditions de l'EHESS

Édition imprimée

Date de publication : 31 décembre 2010

Pagination : 9-242

ISBN : 9782713223013

ISSN : 0335-5985

Référence électronique

François Weiser, "Melissa Wilde, Vatican II: a sociological analysis of religious change », Archives de sciences sociales des religions [En ligne], 152 I octobre-décembre 2010, document 152-119, mis en ligne le 06 mai 2011, consulté le 21 septembre 2020. URL : http://journals.openedition.org/assr/22097 ; DOI : https://doi.org/10.4000/assr.22097

Ce document a été généré automatiquement le 21 septembre 2020

(C) Archives de sciences sociales des religions 


\section{Melissa Wilde, Vatican II: a sociological analysis of religious change}

Princeton, Princeton University Press, 2007, 224 p.

\section{François Weiser}

\section{RÉFÉRENCE}

Melissa Wilde, Vatican II: a sociological analysis of religious change, Princeton, Princeton University Press, 2007, 224 p.

1 Avec ce premier ouvrage, Vatican II : une analyse sociologique du changement religieux, paru en 2007 à Princeton, et non encore traduit en France, Melissa Wilde, professeur à l'université de Pennsylvanie, propose à la réflexion des pages à l'écriture concise et au style alerte. Au croisement de la sociologie religieuse et de la sociologie des organisations, l'auteure vise à rendre compte des changements intervenus au sein de l'Église catholique romaine dans le contexte singulier de la réunion conciliaire tenue entre octobre 1962 et décembre 1965. L'ouvrage se divise très clairement en deux parties, comprenant chacune trois chapitres. Une des rares sociologues, depuis Rock Caporale (Vatican II: the last of the Councils, Baltimore, 1964) dans les années soixante, à entreprendre une telle approche du Concile, M. Wilde innove par les matériaux rassemblés, en particulier les archives du Vatican, qu'elle a pu exploiter pour son étude des votes des quelque trois mille pères conciliaires, sur l'ensemble des quatre sessions du Concile.

2 La première partie de l'ouvrage progresse méthodiquement, à partir de trois questions. Compte tenu du rôle prépondérant joué par la Curie romaine au début du Concile et dans la période qui le précède, rôle de gardien du passé et de verrouillage des textes préparatoires, comment des changements ont-ils pu avoir lieu? Pour construire une sociologie des événements - expression peu claire d'« eventful sociology »-, sans doute 
en ce qu'elle s'attache à une narration, M. Wilde analyse d'abord l'événement conciliaire.

3 Une "chaîne d'événements" (Wilde s'appuie sur la définition d'un événement par William Sewell, 1996, «Historical events as transformation of structures: inventing revolution at the Bastille », Theory and society 25-6, pp. 841-881, cité p. 14), inaugurée au premier matin du Concile, et s'étendant sur l'ensemble de la première session (octobredécembre 1962) aurait conduit à un "coup d'Eglise ", selon l'expression de Joseph Komonchak (12 février 1999, "Convening Vatican II: John XXIII calls for a Council», Commonweal, pp. 10-12). Ainsi se serait produit quasi-instantanément dans l'assemblée une inversion des groupes de majorité (Curie, puis partisans du changement) / minorité (réformateurs, puis tenants d'une doctrine traditionnelle de l'Église comme forteresse assiégée par la modernité), ou, en tout cas, la prise de conscience du rapport réel des forces entre les participants. L'effet de cette prise de conscience est l'entrée dans un état d' "effervescence collective ", en termes durkheimiens, ou, en termes religieux, de "confiance en l'Esprit saint ", évoqué comme celui qui guide et légitime les changements dans l'Église. Ce bouleversement dans la représentation des changements possibles inaugure une série de basculements: le passage, sur le plan culturel, d'une identité ecclésiale perçue comme autorité centrale enseignante à celle d'une société instituante, le peuple de Dieu, et cet autre passage, sur le plan du pouvoir, d'un gouvernement romain à un gouvernement collégial (ne serait-ce que sur la durée du Concile), avec l'institutionnalisation et la valorisation concrète des conférences épiscopales. L'ensemble de ces éléments a fait du Concile, au lieu d'un parlement docile validant des documents curiaux, une véritable instance de débat et de changement.

Puisque des changements ont été rendus possibles, comment expliquer que certains se soient réalisés, et d'autres non? C'est ce que $\mathrm{M}$. Wilde appelle dans son deuxième chapitre la question comparative : qui a voulu quoi, et pourquoi ? Ce chapitre présente certainement les éléments les plus originaux de cette étude. Par l'analyse des votes des pères conciliaires, en particulier quand il s'est agi de dégager une tendance progressiste et une tendance conservatrice (à propos notamment des sources de la révélation, et de la question mariale), l'auteure peut construire une typologie des ensembles épiscopaux. Les priorités de ces groupes, ou stratégies organisationnelles, sont largement déterminées par le "champ organisationnel» dans lequel ces épiscopats sont acteurs. Selon M. Wilde, on peut distinguer quatre champs, ou espaces, en fonction des variables «diversité religieuse » et «stabilité religieuse » : les espaces monopolistiques, les espaces en crise, les espaces émergents et les espaces stables (tableau p. 48). Au passage, on retiendra de cette construction qu'elle a le grand mérite de souligner les limites des catégories de "progressistes » et de "conservateurs », en tout cas de les affiner: toujours relatives à un champ organisationnel différent, ces catégories renvoient en définitive à des réalités plus complexes, qu'une opposition binaire ne permet pas d'atteindre. Une telle typologie rappelle opportunément à quel point les positions prises par les individus, comme par les groupes, ne se comprennent pas en dehors du tissu singulier dont elles sont nées.

5 Reprenant à son compte ce qu'elle considère comme un nouveau paradigme en sociologie des religions, la théorie de la compétition institutionnelle (ou théorie de l'offre, importée des théories économistes), l'auteure en montre les limites: si la compétition entre instances religieuses peut éclairer sur les choix des dirigeants de s'ouvrir au changement, elle ne dit rien sur la nature des changements consentis, ou 
décidés. En particulier pour le groupe, central selon elle dans l'évolution du Concile, d'Europe du Nord et d'Amérique du Nord, comment expliquer qu'il s'applique davantage à améliorer les relations avec son groupe concurrent (les Églises protestantes) qu'à satisfaire aux demandes de ses membres ? C'est parce que le moteur principal du Concile, pour ce groupe influent, serait la question de la légitimité par rapport à leur champ organisationnel. Puisant aux études sur la sociologie des institutions (DiMaggio, Powell et Fligstein en particulier), M. Wilde insiste sur l'homogénéisation des comportements des dirigeants dans des champs organisationnels bien structurés, là où la stabilité et l'entente, y compris par le dialogue, sont préférées à la compétition à outrance. En d'autres termes, pour les épiscopats d'Europe du Nord, dans l'environnement religieux stable qui est le leur, la priorité n'est pas de tenir compte d'abord de la situation des croyants à l'intérieur de leur espace religieux, mais de trouver les termes d'une légitimité accrue par rapport aux dirigeants des autres religions, en l'occurrence les protestants. Évoluant dans des espaces où le religieux est largement structuré et stable, les évêques européens auraient été conscients, plus que leurs collègues, d'avoir un intérêt commun avec les responsables chrétiens des autres dénominations.

6 Ce résultat conduit l'auteure à sa troisième et dernière question : compte tenu des divisions initiales créées par la question œcuménique, et de la variété des situations organisationnelles des évêques, comment les dirigeants des Églises progressistes d'Europe du Nord et d'Amérique du Nord ont-ils pu et su développer des positions de compromis rassemblant les voix d'une majorité d'évêques? C'est grâce à leur organisation pendant le Concile. À la fois plus poussée et plus flexible que celle des évêques conservateurs (en situation monopolistique), leur structuration en réseau leur a permis de dégager, en fonction des textes, des majorités, s'associant les évêques latino-américains et ceux d'Afrique et d'Asie. Cette différence d'organisation procède elle-même d'une différence culturelle majeure : la perception qu'avaient ces évêques de la notion même d'autorité dans l'Église. Une telle organisation a vu ses effets démultipliés par l'adéquation manifeste entre une structure collégiale active, fondée sur la recherche du consensus par la base, et la nature de l'événement conciliaire comme moment de débat, de propositions et de contre-propositions : la conférence des vingt-deux, sous-groupe d'évêques réunis à la Domus Mariae, a été capable, par le biais des conférences épiscopales, de communiquer efficacement avec près de mille neuf cents évêques, sur une base hebdomadaire (schéma p. 65). Face à cette culture du dialogue, la conception de l'autorité comme pouvoir central incapable de débat était portée par d'autres évêques, et cela au nom même de la primauté pontificale. Mais ces évêques, et notamment les plus conservateurs, ont été isolés, d'autant plus qu'ils étaient surpris par un pape (Jean XXIII) qui les renvoyait de fait à leur responsabilité : aux évêques réunis d'élaborer eux-mêmes les textes dont l'Église avait besoin pour son " aggiornamento ", y compris sur la collégialité épiscopale comme pendant nécessaire à l'infaillibilité pontificale.

7 Après ces analyses des ressorts du changement religieux à l'intérieur des mécanismes institutionnels, la seconde partie s'engage, par le biais d'études de cas, à donner une épaisseur historique à ces hypothèses. Sont ainsi passés en revue, sur la durée du Concile, d'une part un texte qui a été adopté, la déclaration sur la liberté religieuse, et d'autre part deux textes qui ont été refusés, l'un sur Marie, l'autre sur le contrôle des naissances. Ces deux derniers cas servent à étayer l'idée que les évêques étaient plus soucieux de légitimité que de satisfaire les membres de leur propre institution : jetant 
toutes leurs forces dans le combat pour que de nouvelles affirmations mariologiques ne séparent pas davantage les protestants et les catholiques, les évêques progressistes ignorent pourtant largement la demande des laïcs concernant la révision de la position de l'Église sur la légitimité des méthodes contraceptives (p. 123).

Dans une démonstration globalement rigoureuse et abondamment documentée, croisant une variété de sources étonnante, aussi bien historiques que sociologiques, c'est peut-être pourtant ce point, maintes fois réitéré, de la priorité accordée à l'amélioration des relations avec les Églises protestantes, qui semble le moins convaincant. Tout se passe, pour l'auteure, comme si la question du dialogue théologique avec les protestants primait, a priori, sur la question du débat théologique à l'intérieur du champ catholique. Or l'exemple français, rangé par M Wilde parmi les « espaces stables » du fait de la séparation des Églises et de l'État, montre pourtant une situation où les débats font rage, d'abord entre auteurs catholiques. On peut se demander si ces auteurs se posent la question de la légitimité de leur discours ad extra, ou tout simplement par rapport à Rome. Ou bien, plus radicalement, par rapport à une autre extériorité, et une autre forme d'instabilité, trop peu abordée, sinon dans le chapitre sur la sexualité : l'extériorité d'une société « indifférente ", où la désaffection du religieux devient une donnée essentielle de l'espace symbolique et social.

Ce travail aurait gagné à ce que ces orientations du Concile, tant par rapport à la modernité que par rapport au protestantisme, soient davantage contextualisées. Il aurait gagné aussi à expliciter davantage la légitimation des choix méthodologiques, au fur et à mesure que ces choix interviennent - même si ceux-ci sont heureusement et fort abondamment documentés dans les notes. Enfin, on relèvera que certaines définitions conciliaires mériteraient d'être nuancées : la collégialité, malgré Vatican II, ne signifie pas que "les évêques réunis disposent de la même autorité que le pape » (pp. 7 et 57); la définition la plus ouverte que les évêques en ont n'oppose pas le pape aux évêques, mais conçoit la collégialité comme l'exercice du lien qui les unit entre eux et avec le pape, primus inter pares. De même, s'il y a bien une nouvelle définition de l'Église proposée par le Concile, en l'occurrence par le biais de l'expression "peuple de Dieu ", cette définition ne s'oppose pas à l'ancienne (l'Église comme autorité hiérarchique), elle s'y ajoute. C'est d'ailleurs, comme souvent pour cette institution, ce qui rend possible la nouveauté : celle-ci n'est pas censée abolir les formules les plus anciennes, mais les compléter. Quoiqu'il en soit, ces réserves ne contredisent en rien la démonstration, réussie par cet ouvrage, de la radicale nouveauté de ce Concile dans l'histoire de l'Église catholique. 